\title{
Pre-analytic phase in molecular biology: criticism and non-compliance management
}

Catia Sias, Loredana Aleo, Stefania Di Filippo, Marco Paterno, Anna Rosa Garbuglia, Maria Rosaria Capobianchi Laboratorio di Virologia - INMI “L.Spallanzani”, Roma

Key words: Pre-analytic phase, non-compliance, extraction, internal control

La fase pre-analitica in biologia molecolare: problematiche e gestione delle non conformità

\section{SUMMARY}

Introduction: During workflow in Laboratories the most delicate and important step is pre-analytic sample treatment because it involves more than one operator of the same structure and often different health services. In fact, the biological materials used for the diagnosis should be collected, sent and properly treated before the analytic phase. Correct methods for collecting and handling biological materials, including guidelines to users of laboratory services, improve performance of Laboratory testing activity. In the pre-analytic phase the operators check sample integrity, and prepare the sample for the subsequent analytic phase: in all these steps monitoring and control of "non- compliance" is crucial.

Methods: During 2007-2008 we created a "non- compliance" check-list, to monitor errors which occurred in different sectors of the preanalytic phase, particularly in the nucleic acid extraction step. These "non-compliances" are analysed to identify and to remove errors, adopting preventive and corrective proceedings. Since 2008 we have been using DNA/RNA internal controls synthesized in our Laboratory. They can be amplified by the same primers and recognized by different probes.

Results: Examination of the "non compliance" check-list for molecular biology investigations shows that the percentage of urine repeat samples decreased from $17 \%$ to $2 \%$ and the percentage of stool repeat samples from $27 \%$ to $2 \%$. Regarding use of internal controls, they allow the assessment of inhibitory factors that can prevent gene amplification.

Conclusions: Monitoring "non-compliance" cases and dividing them by typology allow us identifying the most frequent causes of incorrect sample handling, as a non optimal procedure of pre-treatment, thus improving the pre-analytic phase. Therefore by monitoring the preanalytic phase we can prevent the introduction of confounding factors that may negatively influence the accuracy of results and their interpretation. Before proceeding to gene amplification, biological samples must be properly purified to eliminate lipids, proteins, polysaccharides and other potential inhibitors of DNA polymerase.

\section{INTRODUZIONE}

Il flusso di lavoro in laboratorio comprende tre fasi: pre-analitica, analitica e post-analitica. Nella fase pre-analitica sono incluse tutte le operazioni, a partire dal prelievo fino all'esecuzione di uno specifico test diagnostico. La fase analitica consiste nell'esecuzione del test. La fase post-analitica riguarda la raccolta, l'elaborazione/interpretazione dei risultati e la stampa del referto. Nel corso degli anni, grazie all'introduzione dell'automazione dei sistemi diagnostici e all'utilizzo di controlli di qualità interni ed esterni, l'errore analitico dei test è notevolmente diminuito, con risultati sempre più precisi, affidabili e ripetibili e una maggiore accuratezza diagnostica. Anche per la fase post-analitica l'errore è diminuito con "l'informatizzazione", ovvero con l'utilizzo di programmi che trasferiscono direttamente i risultati dagli strumenti ove sono eseguiti i test al programma gestionale del laboratorio, ove il dato, prima della refertazione, può essere valutato con il supporto di una sintesi storica, per la verifica di eventuali incongruenze. La fase pre-analitica rimane a tutt'oggi una delle fasi più delicate e importanti del flusso di lavoro in laboratorio, essendo completamente manuale. Questa fase pertanto presenta una maggiore incidenza di errori, che influenzano in maniera significativa il risultato finale di un test.

Nella fase pre-analitica si ha il coinvolgimento di più operatori appartenenti a strutture sanitarie differenti. Infatti i materiali biologici utilizzati per le diagnosi devono essere raccolti (infermiere), inviati (corriere) e sottoposti a un idoneo pretrattamento prima di essere utilizzati nei test diagnostici (personale tecnico). L'elaborazione di accurate procedure di raccolta e trattamento dei materiali biologici e la loro comunicazione a chi prepara e invia i campioni garantiscono un'adeguata conservazione dell'integrità clinica e biologica dei medesimi, contribuendo in modo determinante all'accuratezza del dato analitico. Il laboratorio di afferenza dei campioni svolge sia il controllo dell'idoneità dei campioni ricevuti, sia la loro preparazione precedente l'avvio delle fasi analitiche: in tutte queste fasi il monitoraggio e la rilevazione delle "non conformità" sono di grande rilevanza.

In questo nostro lavoro descriviamo la gestione delle "non conformità" nella fase pre-analitica, con particolare riferimento allo step dell'estrazione degli acidi nucleici.

\section{MATERIALI E METODI}

Per il monitoraggio di eventuali inadempienze nella gestione della fase pre-analitica, nell'ambito del sistema di qualità UNI EN ISO9001:2000, abbiamo creato la scheda delle "non-conformità" (Figura I), destinata alla registrazione degli errori della fase pre-analitica rilevati durante l'accettazione e la successiva fase di preparazione dei campioni.

La fase dell'estrazione degli acidi nucleici dei campioni clinici per indagini molecolari appare affetta da maggiore probabilità di errore e la sua inadeguatezza è cruciale per l'attendibilità del risultato finale. Nell'apposita sezione del laboratorio arrivano ogni giorno prelievi sia dai reparti interni che da istituzioni esterne. Ciò comporta la necessità di verificare l'idoneità del prelievo, ad esempio la giusta tipologia di campione biologico e la sua corrispondenza per l'esame richiesto. Nell'attività di routine la preparazione dei campioni viene eseguita ogni giorno da un operatore differente; la tipologia dei campioni è molto varia e ogni campione richiede una differente preparazione. Per questo motivo è stata elaborata una guida pratica per il trattamento e la conservazione dei campioni da sottoporre a estrazione. In essa è descritto in dettaglio il trattamento dei diversi campioni biologici come plasma, sangue, urine, tamponi vaginali o di altro tipo. Considerato il numero di campioni che pervengono nel nostro laboratorio, è stato scelto un sistema di estrazione automatico che permette la lavorazione di un elevato numero di campioni per seduta (32-96) con una minima necessità di intervento da parte dell'operatore. Lo strumento può emettere una lista di lavoro in base alle posizioni di caricamento, facilitando la rintracciabilità dell'eluato. Può inoltre estrarre

\section{Corresponding author: Catia Sias}

c/o Laboratorio di Virologia, INMI "Lazzaro Spallanzani"

00I49 Roma - Via Portuense, 292 - Tel: 06/55 I 70652; Fax: 06/5594555

E-mail: catia.sias@inmi.it 
contemporaneamente DNA ed RNA dallo stesso campione partendo anche da matrici biologiche di diversa natura (siero, sangue, urine..). Pur essendo lo strumento validato con “Conformité Europeenne" (CE) per l'estrazione degli acidi nucleici di plasma siero e sangue, è in effetti possibile estrarre contemporaneamente altri tipi di campioni biologici mediante una diversificazione di pre-trattamento.

Nel 2007-2008 sono stati annotati nelle nostre schede "di non conformità di settore" tutti i campioni di acidi nucleici estratti non risultati adeguati per test di amplificazione genica. Dopo 6 mesi di monitoraggio, abbiamo notato che urine, feci e tamponi vaginali rappresentavano le matrici biologiche con maggior numero di "non conformità". Pertanto abbiamo introdotto dei cambiamenti nel processo di estrazione aumentando il numero di lavaggi e variando il pre-trattamento del campione.

Nel primo semestre 2007 le urine venivano solo stoccate e poi avviate alla fase di estrazione senza alcun pre-trattamento. Dopo il monitoraggio delle "non-conformità", è stato introdotto un pre-trattamento con il buffer di lisi AL (Qiagen) e proteasi, con incubazione del campione a $56^{\circ} \mathrm{C}$ per $10 \mathrm{~min}$. Per i campioni di feci e per i tamponi, sono stati invece aumentati da 2 a 4 i lavaggi dell'estrattore e, per i soli campioni di feci, si è proceduto alla doppia estrazione.

Per valutare l'idoneità delle nostre procedure di estrazione, abbiamo per anni utilizzato l'amplificazione di una regione della ß-globina (house-keeping gene) e, in campioni privi di cellule o con scarsa cellularità (liquor, plasma) abbiamo aggiunto un plasmide contenente una regione del gene della ß-globina. In tal modo è stato possibile saggiare tutti i campioni con lo stesso set di primers e quindi valutarne l'idoneità per la ricerca di virus a DNA. Per valutare l'idoneità del campione per la ricerca di virus a RNA, abbiamo aggiunto ad ogni singolo campione il fago MS2, retro- trascritto e amplificato. In entrambi i casi la rivelazione del prodotto di amplificazione è stata fatta con gel di agarosio.

Nel 2008 abbiamo introdotto dei controlli interni (IC), da noi sintetizzati, uno a DNA e l'altro a RNA. Questi frammenti sono stati sintetizzati in modo da avere una omologia minima con sequenze depositate in GenBank. Come si può vedere nella Figura II, essi vengono amplificati con lo stesso set di primers (1). Questi controlli interni sono aggiunti in numero di copie note al buffer di lisi utilizzato dall'estrattore automatico, dunque ripartiti in maniera uniforme in tutti i campioni da estrarre. Cinque microlitri di eluato sono amplificati in real-time, utilizzando una sonda specifica per il controllo interno a DNA marcata al 5' con VIC e una sonda specifica per il controllo interno ad RNA marcata al 5' con FAM.

Il range di accettabilità dei cicli soglia o threshold cycles (Ct) è di 27-30. Per valori di $\mathrm{Ct}>30$ il campione è considerato "inibito", quindi si deve ripetere l'estrazione e non si prosegue nelle indagini molecolari. In tre ore si hanno i risultati sull'idoneità di tutti i campioni di una seduta.

\section{RISULTATI}

I campioni “non idonei” per le indagini di biologia molecolare sono stati individuati per tipologia nell'arco dei due semestri dell'anno 2007 e nell'anno 2008. È da notare che il maggior numero di campioni "non idonei" ha riguardato le urine (Tabella 1). Dopo l'introduzione della procedura di pre-trattamento, la percentuale di "non idoneità" è scesa dal $17 \%$ (primo semestre 2007) al 2\% (secondo semestre del 2007), mantenendosi stabile nell'anno 2008.

I campioni di feci e i tamponi, nonostante il pre-trattamento con proteasi, hanno continuato a dare problemi di "non idoneità", presumibilmente i primi per la presenza di lipidi che interferiscono con le polimerasi e i secondi per la presenza di sostanze fortemente inibenti nell'area di flogosi ove viene effettuato il prelievo. Abbiamo quindi modificato il numero dei lavaggi dell'estrattore, aumentandoli da 2 a 4. Questo intervento correttivo è risultato sufficiente per i tamponi, con una percentuale di campioni ripetuti del $2 \%$ nei due semestri del 2007, scesa poi all’1\% nell'anno 2008 (Tabella 2).
Invece, con il solo aumento dei lavaggi i campioni di feci "non idonei"sono passati dal 27\% del primo semestre 2007 al $18 \%$ del secondo semestre, ma per una riduzione al $2 \%$ nell'anno 2008 si è dovuta adottare come intervento correttivo la doppia estrazione del campione (Tabella 3).

Per quanto riguarda la valutazione della qualità dell'estratto è necessario sottolineare che l'impiego della ß-globina come controllo interno non fornisce risultati accurati. Infatti il gene della ß-globina è endogeno e presente in alto numero di copie nei campioni ad elevata cellularità: in quanto tale può mascherare la presenza di fattori inibenti. Inoltre il fago MS2 deve essere retro-trascritto, il cDNA amplificato e i prodotti di amplificazione rivelati su gel di agarosio, con un impiego minimo di 2 giorni lavorativi.

Il ricorso a controlli interni - sia a RNA che a DNA - coestratti con i campioni permette una valutazione più affidabile della qualità dell'estratto e garantisce l'accuratezza delle successive indagini molecolari, sia per i virus ad RNA che per quelli a DNA. Inoltre la valutazione quantitativa riferita ai cicli soglia consente di verificare con esattezza la presenza di fattori inibenti. Questo nuovo tipo di valutazione dell'idoneità delle procedure di estrazione ci ha permesso anche di ridurre i tempi di risposta per i campioni da saggiare in urgenza, come i liquor.

Tabella I. Percentuale degli esami ripetuti per la tipologia "campione urine".

\begin{tabular}{lccc}
\multicolumn{5}{c}{ URINE } \\
\hline Periodo & Esami ripetuti & Esami effettuati & $\%$ \\
\hline $1^{\circ}$ sem. 2007 & 15 & 89 & 17 \\
\hline $2^{\circ}$ sem. 2007 & 2 & 94 & 2 \\
\hline Anno 2008 & 35 & 1502 & 2 \\
\hline
\end{tabular}

Tabella 2. Percentuale degli esami ripetuti per la tipologia "tampone".

\begin{tabular}{lccc}
\hline \multicolumn{5}{c}{ TAMPONI } \\
\hline Periodo & Esami ripetuti & Esami effettuati & $\%$ \\
\hline I $^{\circ}$ sem. 2007 & 7 & 308 & 2 \\
\hline $2^{\circ}$ sem. 2007 & 7 & 318 & 2 \\
\hline Anno 2008 & 6 & 599 & 1 \\
\hline
\end{tabular}

Tabella 3. Percentuale degli esami ripetuti per la tipologia "campione feci".

\begin{tabular}{lccc}
\hline \multicolumn{5}{c}{ FECI } \\
\hline Periodo & Esami ripetuti & Esami effettuati & $\%$ \\
\hline$I^{\circ}$ sem. 2007 & 8 & 30 & 27 \\
\hline $2^{\circ}$ sem. 2007 & 5 & 28 & 18 \\
\hline Anno 2008 & 1 & 56 & 2 \\
\hline
\end{tabular}

\section{DISCUSSIONE}

I metodi molecolari rivestono un ruolo importante nella diagnosi delle malattie infettive per la loro alta sensibilità e specificità. Tra i differenti step che concorrono all' affidabilità di un test di diagnostica molecolare, l'estrazione degli acidi nucleici DNA/RNA è quello che presenta maggiore criticità. Pertanto, prima di procedere all'amplificazione genica, i campioni biologici (sangue, urine, tamponi, feci...) devono non soltanto essere preparati per l'estrazione e la concentrazione degli acidi nucleici, ma anche adeguatamente purificati da lipidi, proteine, polisaccaridi ed altri potenziali inibitori della DNA polimerasi.

L'estrazione degli acidi nucleici consiste nel loro isolamento e nella purificazione e concentrazione del prodotto-eluato. Attualmente sono disponibili numerosi sistemi commerciali molto più standardizzati riproducibili e rapidi dei metodi inhouse. Tuttavia, con tali sistemi e i relativi kit non si è in grado di estrarre con la stessa efficienza gli acidi nucleici da tutte le matrici biologiche. Per rendere il campione idoneo all'estrazione di acidi nucleici in sistemi automatici è necessario effettuare un adeguato pre-trattamento. Oltre a ciò, nell'attività di routine, è importante accertare l'idoneità del campione per i saggi molecolari onde evitare risultati falsi negativi. 


\section{Scheda non conformità}

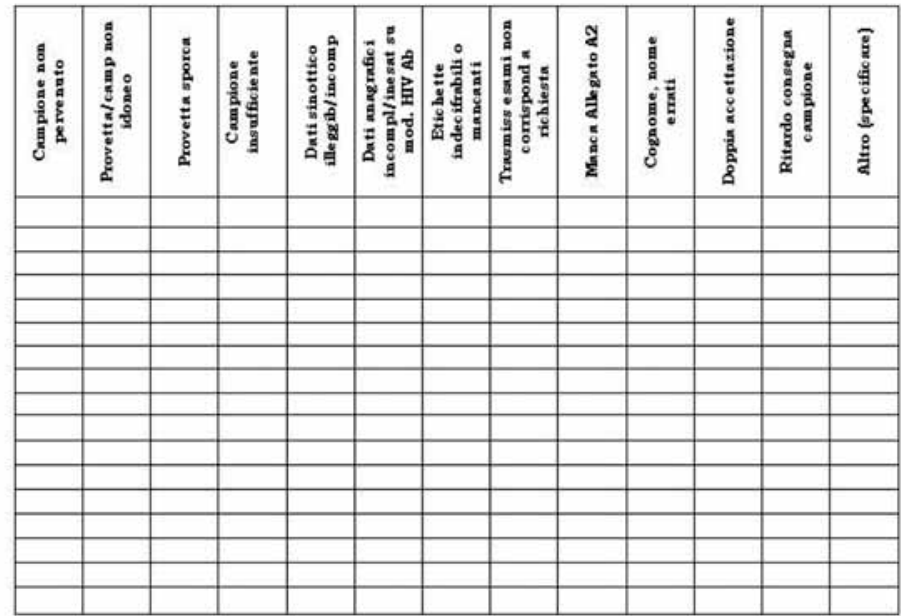

\section{ISO 9001: 2000}

Figura I. Scheda della "non conformità" con le varie tipologie di errore riscontrabili nella fase pre-analitica.

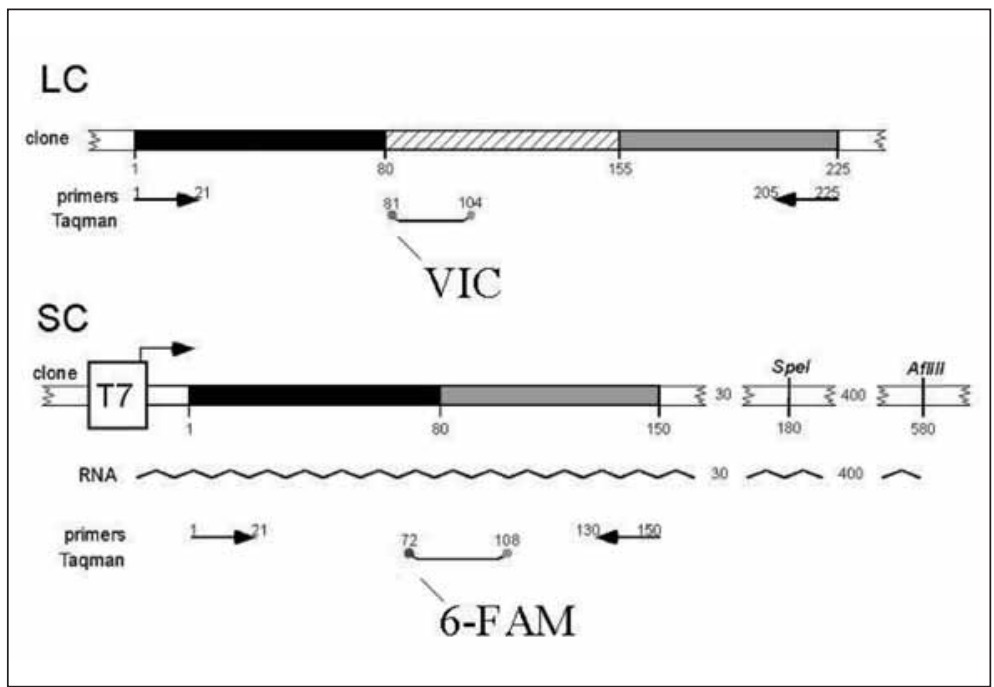

Figura II. Costrutti sintetici utilizzati come controlli interni a DNA e ad RNA che riconoscono sonde specifiche marcate in 5' rispettivamente con VIC e con FAM ercentuale.

In questo nostro studio abbiamo voluto verificare come le modifiche della fase di pre-trattamento e l'aumento dei lavaggi durante la fase di estrazione possano influire sull'efficienza di estrazione di alcune tipologie di campioni. Inoltre, come un sistema Real-time per valutare l'idoneità degli estratti permetta di individuare con maggior precisione eventuali inibitori rimasti nell'eluato.

L'introduzione di due controlli interni, uno a RNA e l'altro a DNA, ci ha permesso di verificare se le sostanze inibitrici siano state rimosse con efficienza, essendo la loro amplificazione in Real-time considerata accettabile quando i valori dei cicli soglia rientrano in un preciso intervallo. Tale sistema consente di rilevare la presenza di fattori inibenti che non sarebbe possibile mettere in evidenza con l'analisi qualitativa su gel di agarosio. La rilevazione di fattori inibenti, anche in modeste quantità, risulta particolarmente importante nei campioni con bassa carica virale, come liquor o lavaggi oculari, ove tracce di inibitori possono dar luogo a risultati errati (falsi negativi). Il numero ancora consistente di campioni di feci risultati “non-idonei” ci spinge peraltro a un’ulteriore ottimizzazione del protocollo di pre-trattamento del campione, anche alla luce di recenti dati di letteratura che suggeriscono una maggiore diluizione del campione e una incubazione a $60^{\circ} \mathrm{C}(2)$.

Per i tamponi, la doppia estrazione dei campioni non idonei potrebbe rappresentare una soluzione ottimale, evitando la richiesta d'invio di un nuovo campione. Sono in allestimento studi comparativi con l'utilizzo di standard internazionali per valutare se tale procedura può provocare la perdita di un target virale presente nel campione in basso numero di copie.

La presenza di problemi di estrazione dei campioni di urine è già stato riportato in letteratura (4).

Nel nostro caso il pre-trattamento con buffer di lisi e proteasi $\mathrm{K}$, comprensivo di una incubazione a $56^{\circ} \mathrm{C}$ e una diluizione del campione, ha migliorato i risultati con una diminuzione dei campioni non idonei dal $17 \%$ al $2 \%$.

È da ricordare che il sistema da noi utilizzato per la preparazione di urine non è conforme alla "In vitro diagnostic (IVD) directive" della CE e quindi modifiche ad hoc devono essere introdotte per il singolo caso al fine di raggiungere risultati più soddisfacenti.

È altresì noto in letteratura che i sistemi, come il nostro, basato sull'estrazione di acidi nucleici mediante colonnine con membrana di silice, possiedono una capacità inferiore ad altri nel rimuovere sostanze inibenti da feci ed urine (es. biglie magnetiche) (3).

Tuttavia, al momento dell'esecuzione dello studio, non esistevano in commercio sistemi automatici a base di biglie magnetiche in grado di effettuare l'estrazione di 32-96 campioni in una singola seduta. I sistemi tuttora esistenti richiedono l'intervento dell'operatore in varie fasi dell'estrazione e permettono di processare un numero inferiore di campioni estraibili per seduta (1-24), condizioni che mal si adattano alla nostra realtà di laboratorio.

Infine ci preme sottolineare l'utilità del Control System Quality nel gestire e monitorare l'attività nei diversi settori coinvolti nella fase pre-analitica.

Con l'introduzione delle schede di "non conformità” è stato possibile monitorare disguidi operativi e attuare azioni correttive finalizzate a un miglioramento dell'organizzazione del lavoro, facilitando l'elaborazione di protocolli per la corretta gestione dei campioni. Attività gestionali queste raccomandate in tutte le linee guida per le buone regole di laboratorio (Guidelines for Good Laboratory Practice) internazionali, volte a migliorare il Servizio Sanitario Pubblico.

\section{BIBLIOGRAFIA}

1. Bartolini B, Garbuglia AR, Horejsh D, et al. Simultaneous control of DNA and RNA processing efficiency using a nucleic acid calibration set. BioTecniques 2007; 42: 452-6.

2. Bolanaki E, Kottaridi C, Dedepsidis E, et al. Direct extraction and molecular characterizazion of enteroviruses genomes for human faecal samples. Molecular and Cellular Probes 2008; 22: 156-61.

3. Petrich A, Mahony J, Chong S, et al. Multicenter Comparison of Nucleic Acid Extraction Methods for Detection of Severe Acute Respiratory Syndrome Coronavirus RNA in Stool Specimens. Journal of Clinical Microbiology 2006; 44: 2681-8.

4. Tang YW, Sefers SE, Li H, et al. Comparative Evaluation of Three Commercial Systems for Nucleic Acid Extraction from Urine Specimens. Journal of Clinical Microbiology 2005; 43: 4830-43. 